\title{
Forward physics results from ATLAS
}

Michele Viti on behalf of the ATLAS collaboration Hadron Structure '11 


\section{Content}

- Overview of the detectors

- Lucid

- ZDC

- MBTS

- ALFA

- Luminosity measurements

- 2 ATLAS results presented

- Inelastic pp cross section

- Rapidity gap cross section 


\section{ATLAS forward detectors}

ATLAS IP

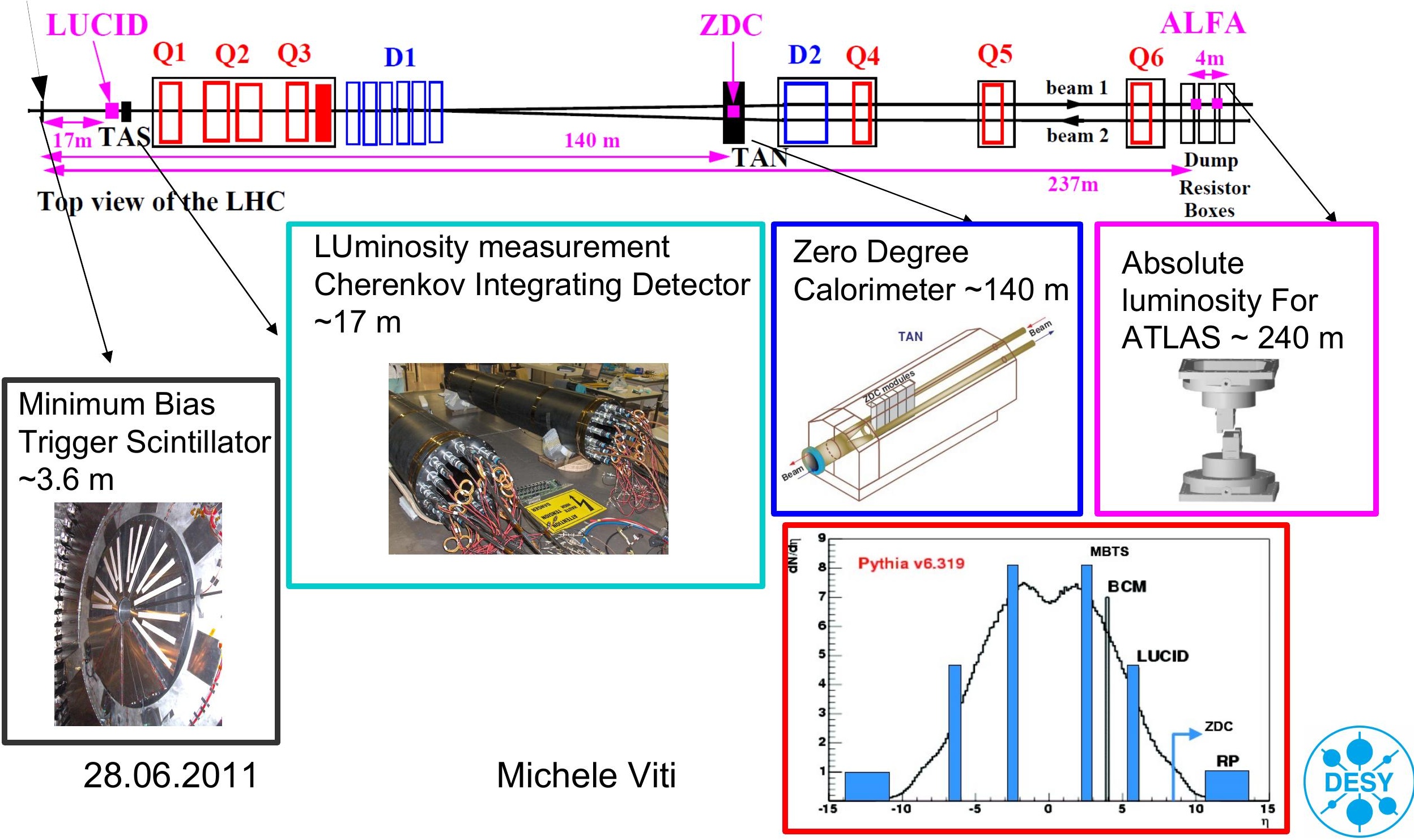




\section{MBTS: Minimum Bias Trigger Scintillator}

- Each side segmented into 2 discs, each disc in $\phi$ in 8 scintillator counters.

- Primary minimum bias trigger for low luminosity (rapidity gap topology).

- Used also for relative luminosity measurement.
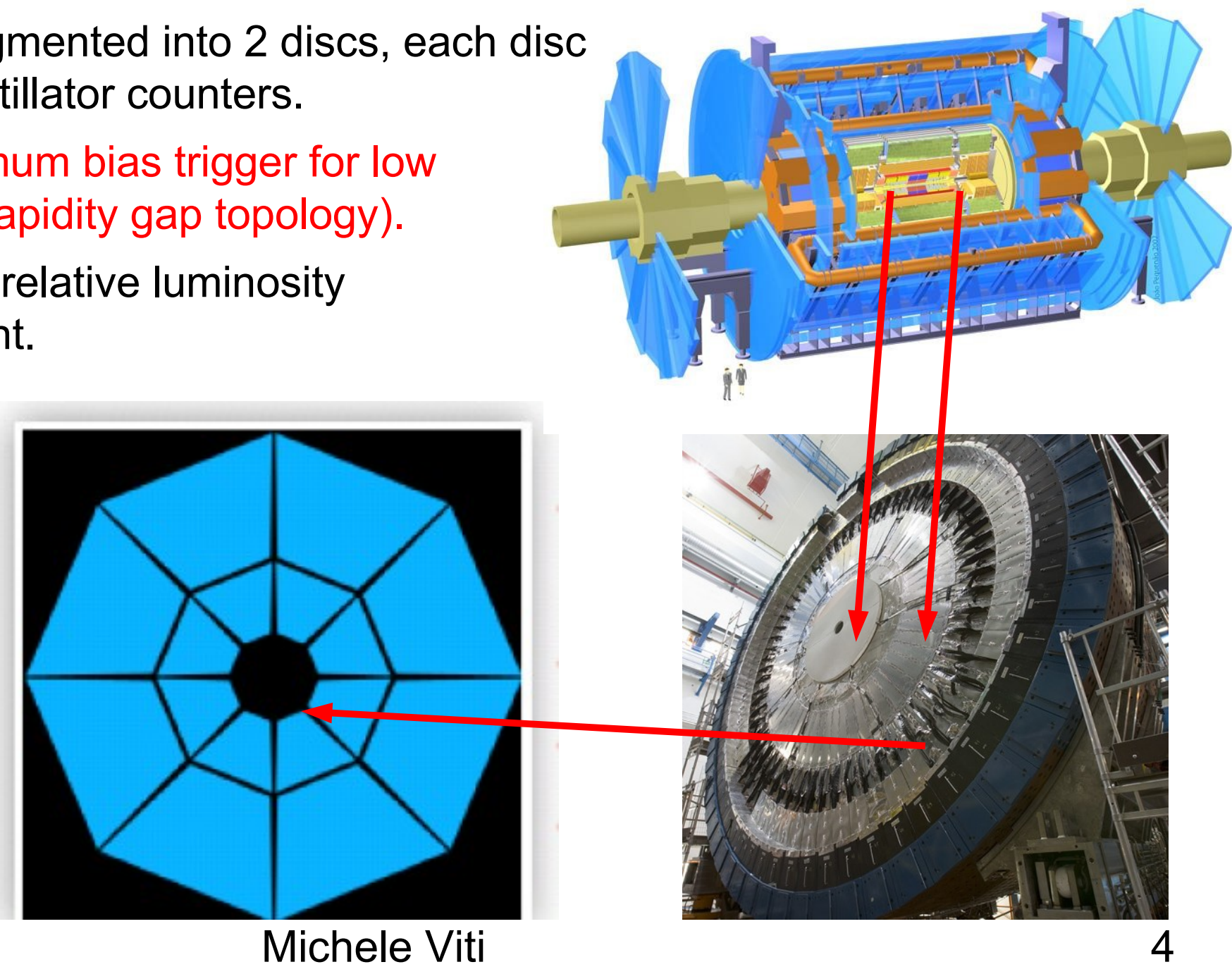


\section{LUCID: LUminosity Cherenkov Integrating Detector}

Forward detector using Cherenkov light.

-2 symmetric detector at $17 \mathrm{~m}$.

-20 tubes filled with Gas.

- Sensitive to charged particle coming from the IP (tubes pointing to the IP). -Fast response (bunch-by-bunch measurement).

- Radiation hardness.

- Online Luminosity monitor. Designed to work from $10^{27} \mathrm{~cm}^{-2} \mathrm{~s}^{-1}$ up to $4 \times 10^{33}$ $\mathrm{cm}^{-2} \mathrm{~s}^{-1}$

-It can provide also minimum bias trigger at high $\eta$.

28.06.2011

Michele Viti
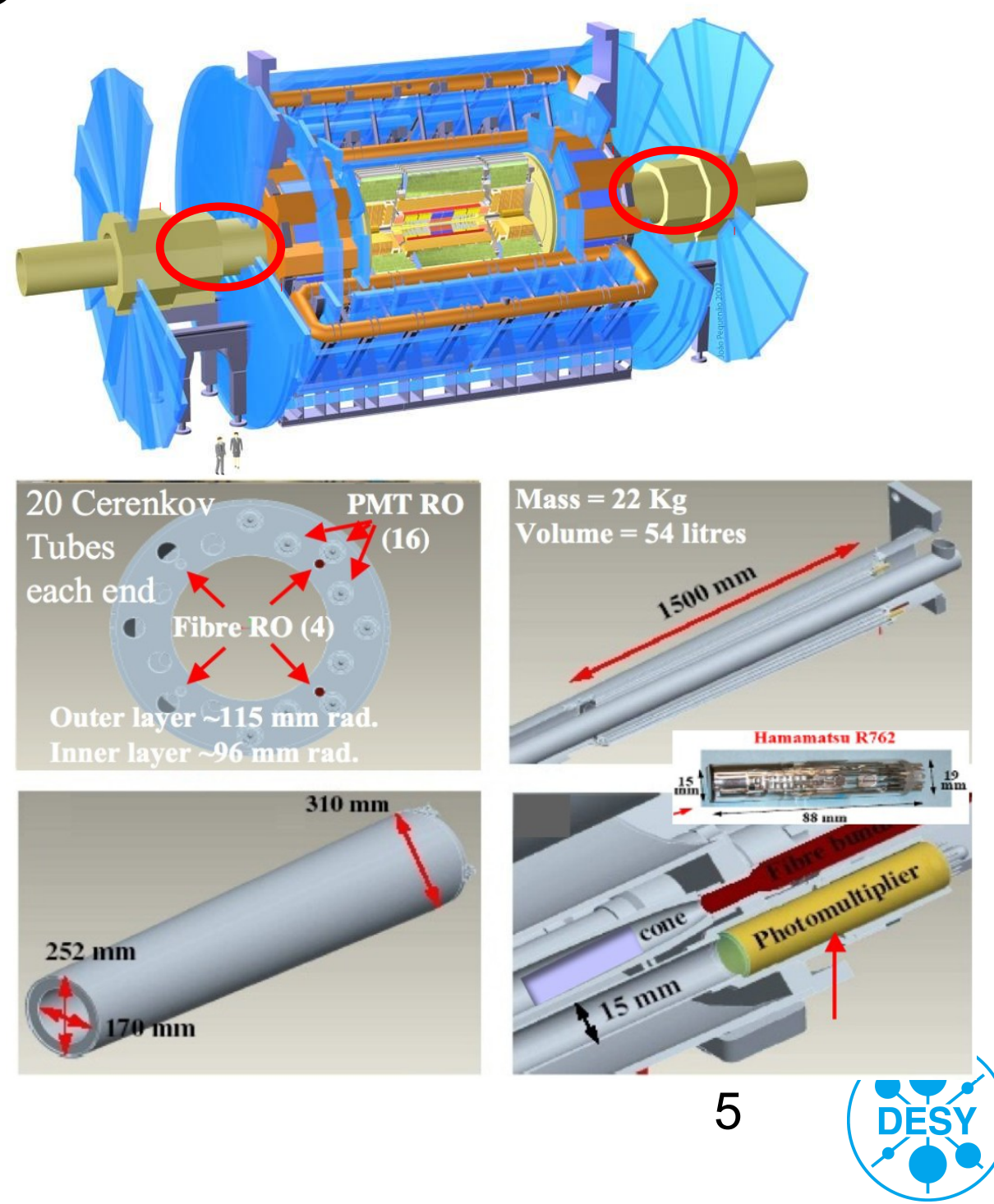


\section{ZDC: Zero Degree Calorimeter}

- 4 modules/arm: 1 EM $\left(29 X_{0}\right), 3 \mathrm{Had}$ $\left(1.14 \lambda_{\text {int }}\right)$, similar in composition.

- Both ZDCs reside in a slot of the TAN Absorber $140 \mathrm{~m}$ from IP.

- Detected neutral particles.

- 11 Tungsten plats

- $+1 \mathrm{~mm}$ quartz rod parallel to beam for position measurement.

- $+1.5 \mathrm{~mm}$ quartz rod for energy measurement.

- Radiation hardness:

- At $10^{33} \mathrm{~cm}^{-2} \mathrm{~s}^{-1}$ it survives for few years.

- At $10^{34} \mathrm{~cm}^{-2} \mathrm{~s}^{-1}$ it survives for few months.
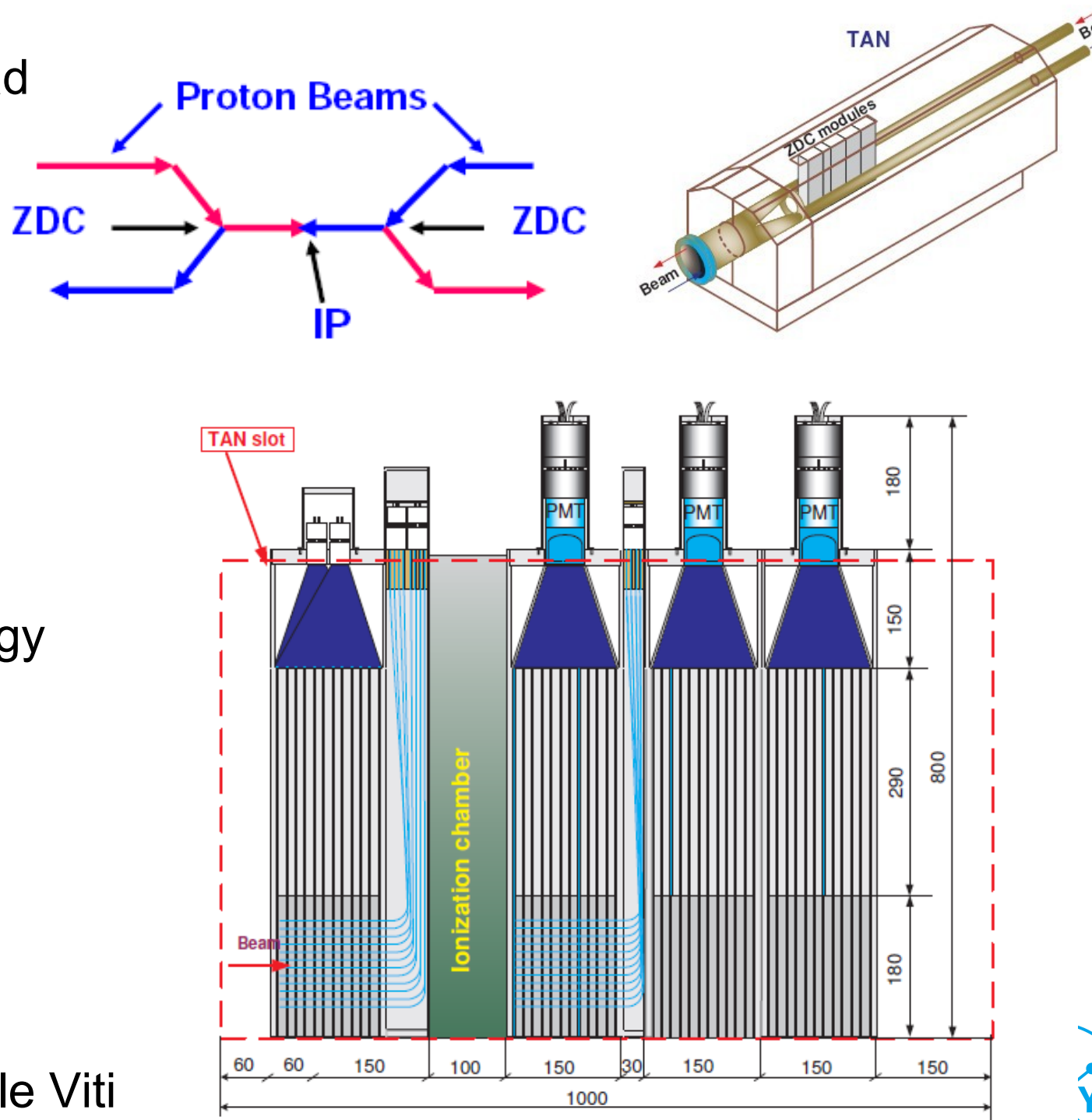


\section{Absolute Luminositv For ATLAS}

- 8 scintillating fiber detectors located in Romans Pots.

- $0.5 \times 0.5 \mathrm{~mm}^{2}$ square fibers arranged in UV geometry.

- 2 Roman Pots (1 up and 1 down) form a station.

- Resolution of 30-40 $\mu \mathrm{m}$ required.

- Determination of the absolute luminosity for ATLAS with an accuracy of $\sim 3 \%$.

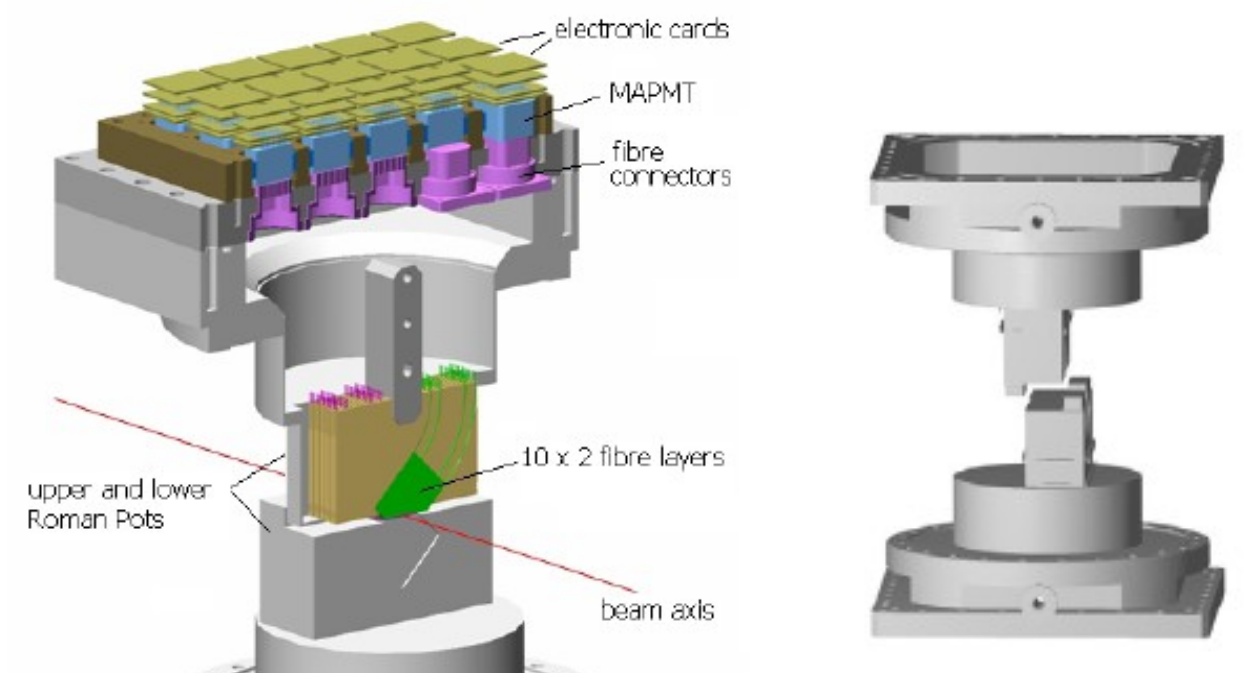

28.06.201

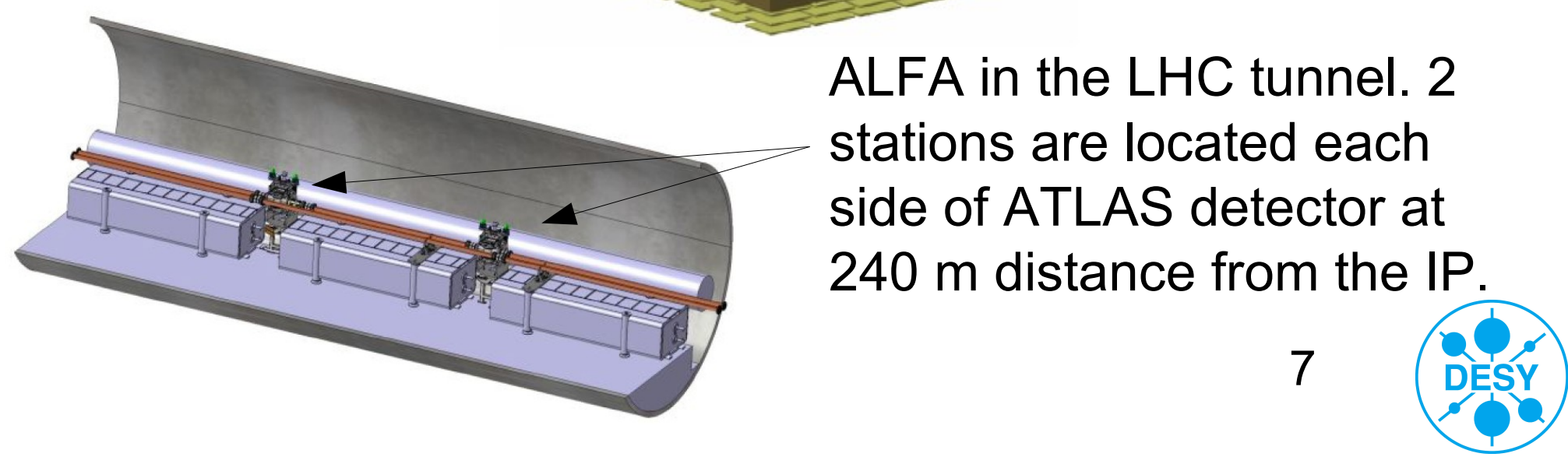




\section{Absolute Luminosity For ATLAS}

- Special optic conditions required

- large value of betatron function at the detector and IP (small emittance).

- $90^{\circ}$ phase advance in the vertical plane.

- negligible dispersion.

- Consequences

- low bunch charge.

- few bunches

$\bullet \rightarrow$ low luminosity $\sim 10^{27} \mathrm{~cm}^{-2} \mathrm{~s}^{-1}$.

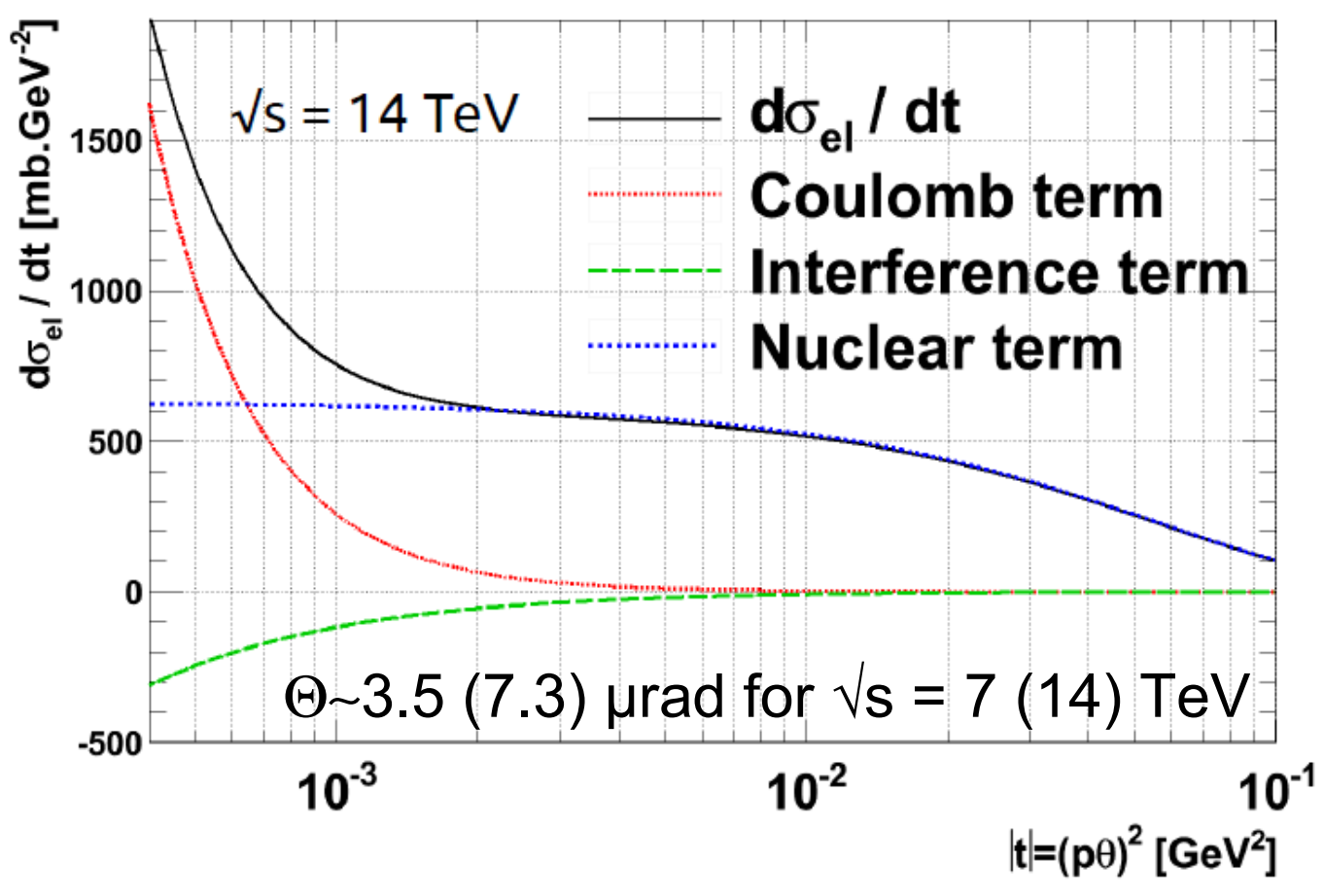

$$
\frac{\mathrm{d} N}{\mathrm{~d} t}=L \pi\left|f_{C}+f_{N}\right|^{2} \approx L t\left|-\frac{2 \alpha}{|t|}+\frac{\left.\sigma_{t o}\right)}{4 \pi}(i+\rho) e^{-b|t| / 2}\right|^{2}
$$

- Measurement of the scattering angle for elastic scattered protons.

- Determination of the absolute luminosity (plus total cross section).

- Calibration of LUCID. 


\section{Luminosity measurement}

-Fundamental parameter for cross section measurement.

-Already the largest contribution to the errors for many measurements.

-At LHC start MC predictions, 20 \% error.

-2010/2011 luminosity determined by machine parameters using the Van der Meer (VdM) scan. An error of $3.4 \%$ was reached in 2010.

-When sufficient statistic will be reached, $\mathrm{W} / \mathrm{Z}$ production will be also used, $\sim 5 \%$ error.

-In the next years, ALFA will provide a luminosity calibration with an error $\sim 3 \%$.

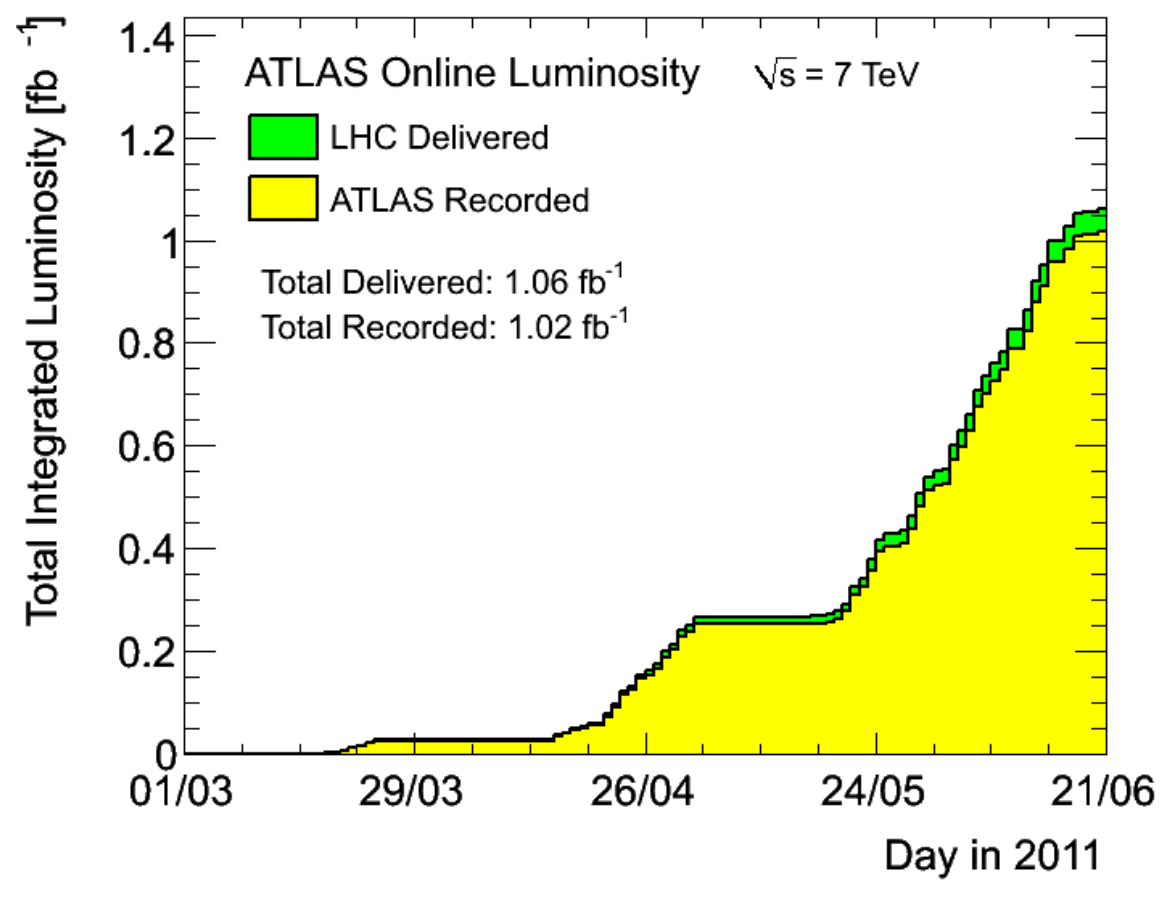

- Recently we reached $1 \mathrm{fb}^{-1}$

- ATLAS efficiency 96\%

- Max peak luminosity $1.26 \times 10^{-33} \mathrm{~cm}^{-2} \mathrm{~s}^{-1}$

- Max bunch. 1092 


\section{Soft QCD/Diffraction}

- Total cross section, (in)elastic cross section, fundamental quantity

_ They cannot be calculated by perturbative QCD.

$-\sigma_{\text {tot }}=\sigma_{\text {el }}+\sigma_{\text {inel }}$

- Diffractive event.

$-\sigma_{\text {inel }}=\sigma_{\mathrm{ND}}+\sigma_{\mathrm{SD}}+\sigma_{\mathrm{DD}}$

_ They represent a large fraction of the inelastic event. ( $30 \%$ inelastic events).

_ In the theory:

- Exchange of the quantum numbers of the vacuum.

- Pomeron exchange in the t-channel

_ In the experiment:

- Large rapidity gap and/or intact protons

$\rightarrow$ low noise and few pile-up events

$\rightarrow$ forward detectors as veto/triggers 


\section{Diffraction}
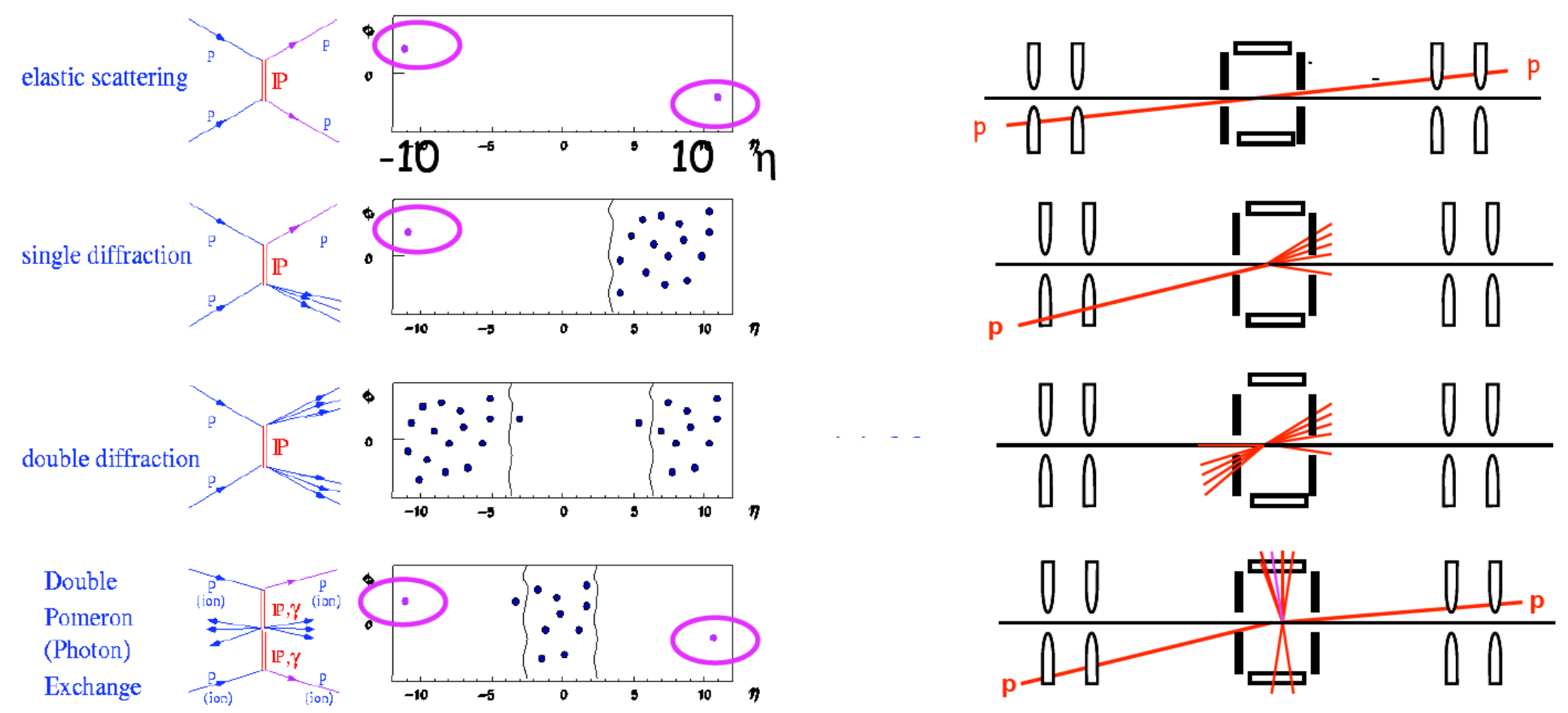

Multi

Pomeron

Exchange
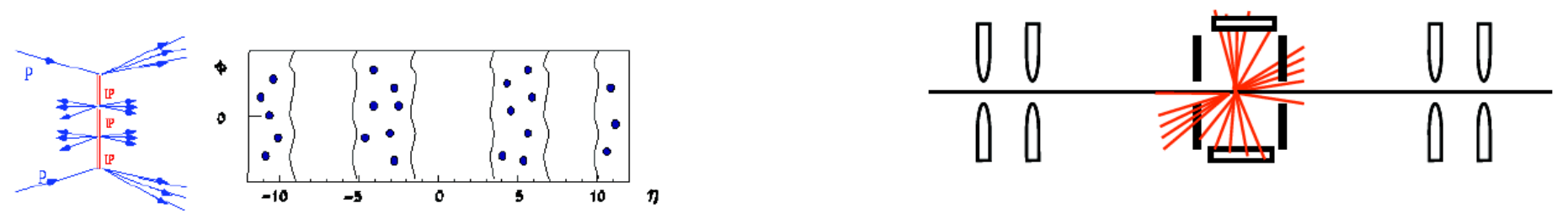

28.06.2011

Michele Viti 


\section{Total Cross Section with ALFA}

-August/September ALFA will perform

together with TOTEM first measurement of

the total cross section.

-Beta function $\sim 90 \mathrm{~m} \rightarrow$ measurement not in the interference region.

-Luminosity needed as input parameter (LUCID+VdM).

- Expected error $<5 \%$. 


\section{Results from ATLAS experiment}

Some results from ATLAS measurements

will be presented

- Measurement of the inelastic pp cross section a $\sqrt{ } \mathrm{s}=7 \mathrm{TeV}$ with the ALTAS detector (ArXiv:1104.0326 [hep-ph])

- Rapidity gap cross sections in pp Interactions at $\sqrt{ } s=7 \mathrm{TeV}$ (ATLAS-CONF-2011-059) 


\section{Inelastic pp cross section}

-Fundamental detector: Minimum Bias Trigger Scintillator.

-Coverage: $2.09<|\eta|<3.84$.

$\rightarrow \xi=\mathrm{M}^{2} / \mathrm{s}>5 \times 10^{-6}, \mathrm{M}$ invariant mass of the dissociated system.

-Trigger: require at least one hit in the MBTS counters.

-Offline selection: require two counter's charge to be above $0.15 \mathrm{pC}$. 


\section{Inelastic pp cross section}

- $\mathrm{R}_{\mathrm{ss}}$, ratio events single side (dominated by SD and DD) and inclusive events.

- $R_{\text {sS }}$ in function of $f_{D}$

(the ratio diffractive/ total inelastic events).

- PYTHIA and PHOJET with different input parameter.

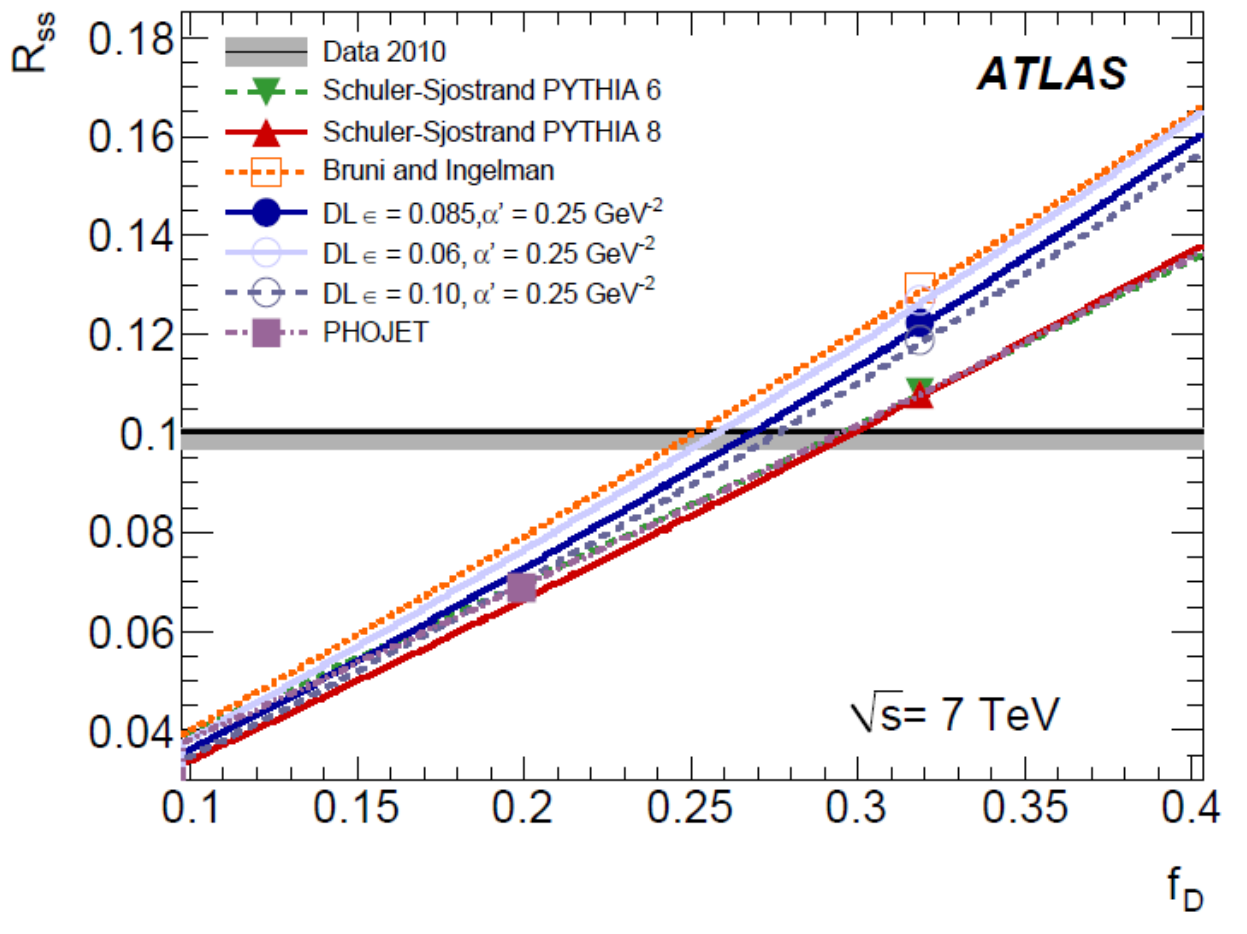

- Results for $f_{D}$ between

$25 \%$ and $30 \%$.

28.06.2011 


\section{Inelastic pp cross section}
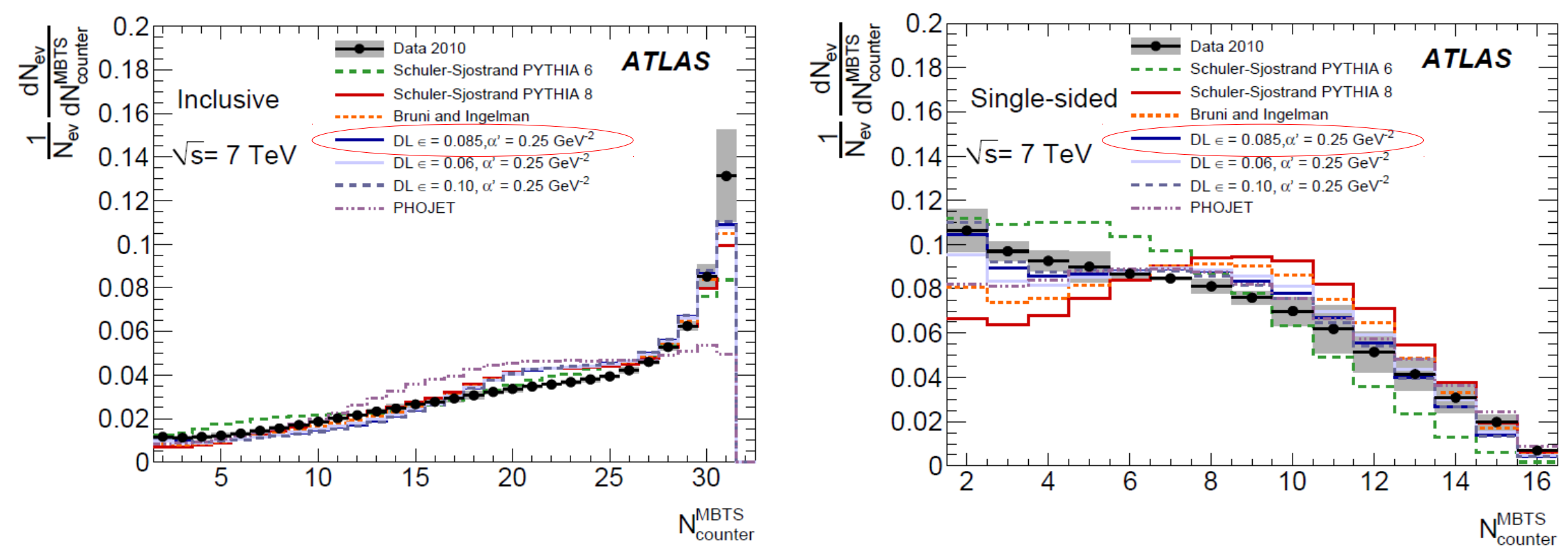

MC validation with data

-MBTS hit multiplicity for the single side and inclusive events

-Pythia based on DL model good description of the single side events $(\rightarrow$ good description of the diffractive part)

-For the inclusive events data lie between the models at low multiplicity (important for the extrapolation).

28.06.2011

Michele Viti

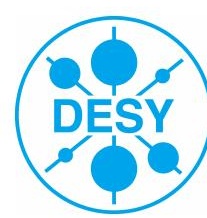




\section{Inelastic pp cross section}

$$
\begin{gathered}
\sigma\left(\xi>5 \cdot 10^{-6}\right)=60.3 \pm 0.05(\text { stat. }) \pm 0.5(\text { sys. }) \pm 2.1(\text { lumi. }) \mathrm{mb} \\
\sigma_{\text {inel }}=69.1 \pm 2.4(\text { exp. }) \pm 6.9(\text { extr. }) \mathrm{mb}
\end{gathered}
$$

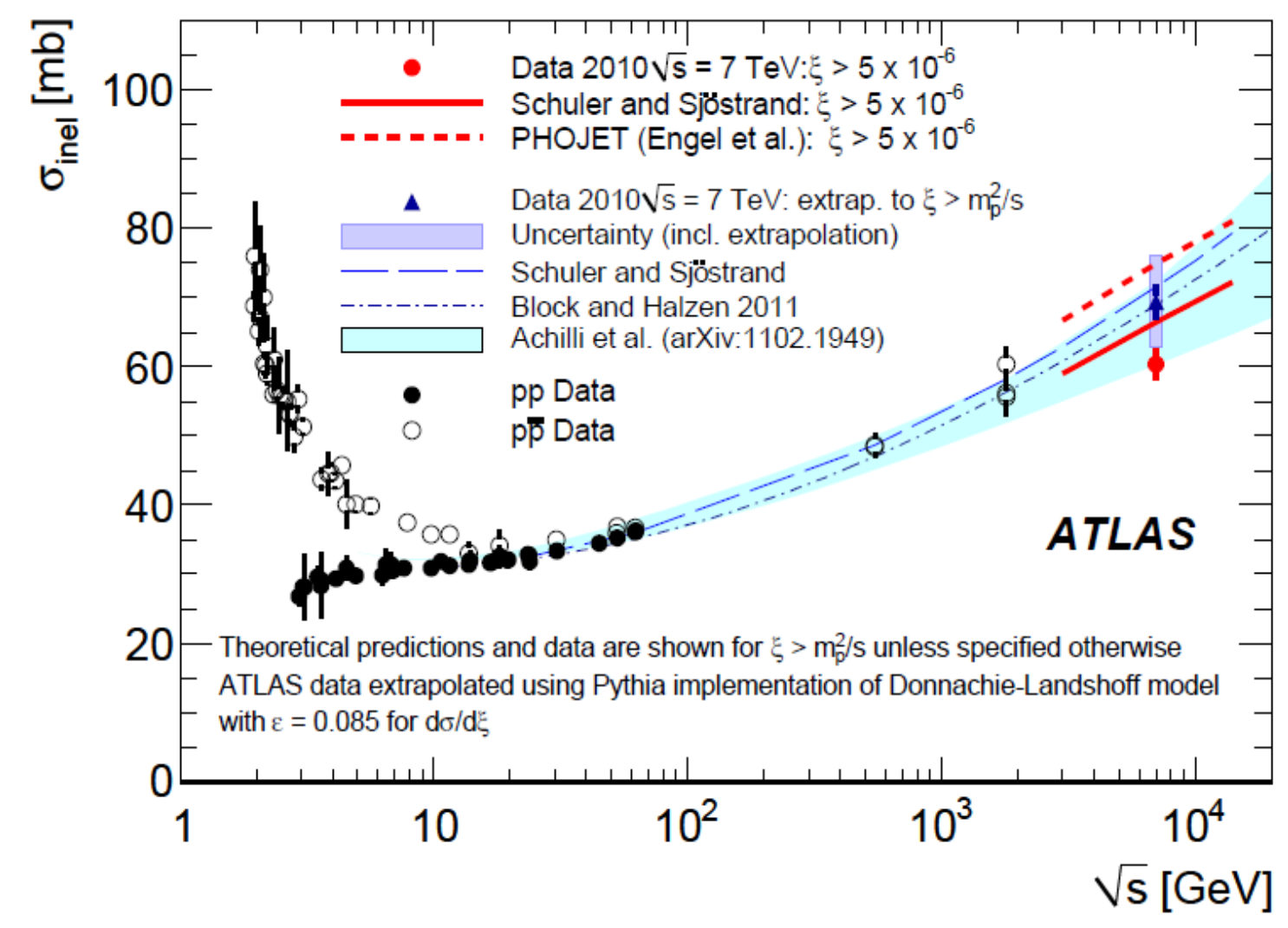




\section{Inelastic pp cross section}

- First measurement from ATLAS of the pp inelastic cross.

- Luminosity dominant experimental error.

- Low-mass diffraction is not measured because the diffractive system is outside the acceptance. This has a large uncertainty (not easy to predict).

- Considering prediction for several model an error of $10 \%$ was given for this extrapolation.

- ALFA can increase the acceptance?

- Consistency with predictions from power law and logarithmic dependences from center-of-mass energy. 


\section{Inelastic pp cross section}

-Possibility to measure this quantity without large extrapolation.

-Integrating the rate we have the elastic cross section.

-With the optical theorem we have the total cross section.

$$
\rightarrow \sigma_{\text {inel }}=\sigma_{\text {tot }}-\sigma_{\text {el }}
$$




\section{Rapidity Gap Cross Section}

- Detector used

- Liquid argon calorimeter $|\eta|<4.9$.

- Inner detector $\mid \eta<2.5$.

- MBTS as trigger.

- Gap Algorithm

- Divide the calorimeter in $\eta$ rings.

- One ring fired if

- At least one track with $p_{T}>200 \mathrm{MeV}$.

- One calorimeter cell above threshold.

- 2 definitions of rapidity gap.

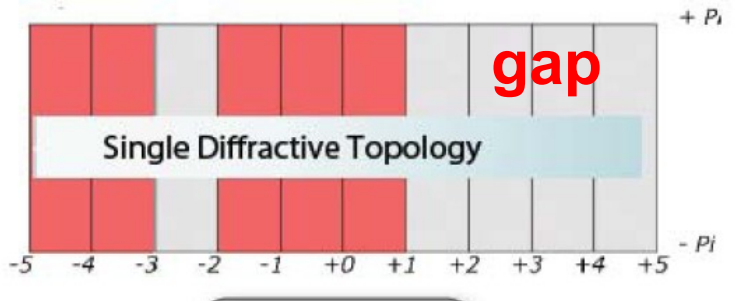

$$
\begin{aligned}
& \Delta \eta=4 \\
& \Delta \eta^{\mathrm{F}}=4 \\
& \eta_{\text {start }}=5
\end{aligned}
$$

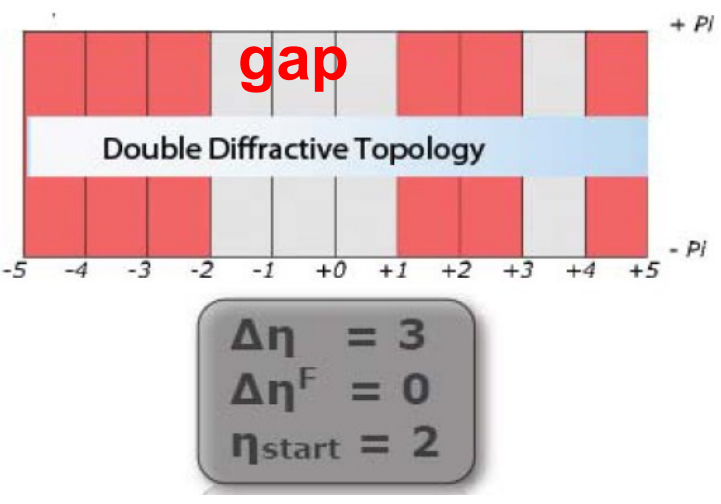

MC Optim. $\{$ - Floating gap: largest consecutive runs of empty rings, parametrized by its size $\Delta \eta$ and $\left|\eta_{\text {start }}\right|$ (double diffraction topology).

Cross section determination
- Forward gap: largest consecutive runs of empty rings, starting from the edge of acceptance $|\eta|=4.9$ (single diffraction topology) with size $\Delta \eta^{F}$ 


\section{Rapidity Gap Cross Section}

$\approx$
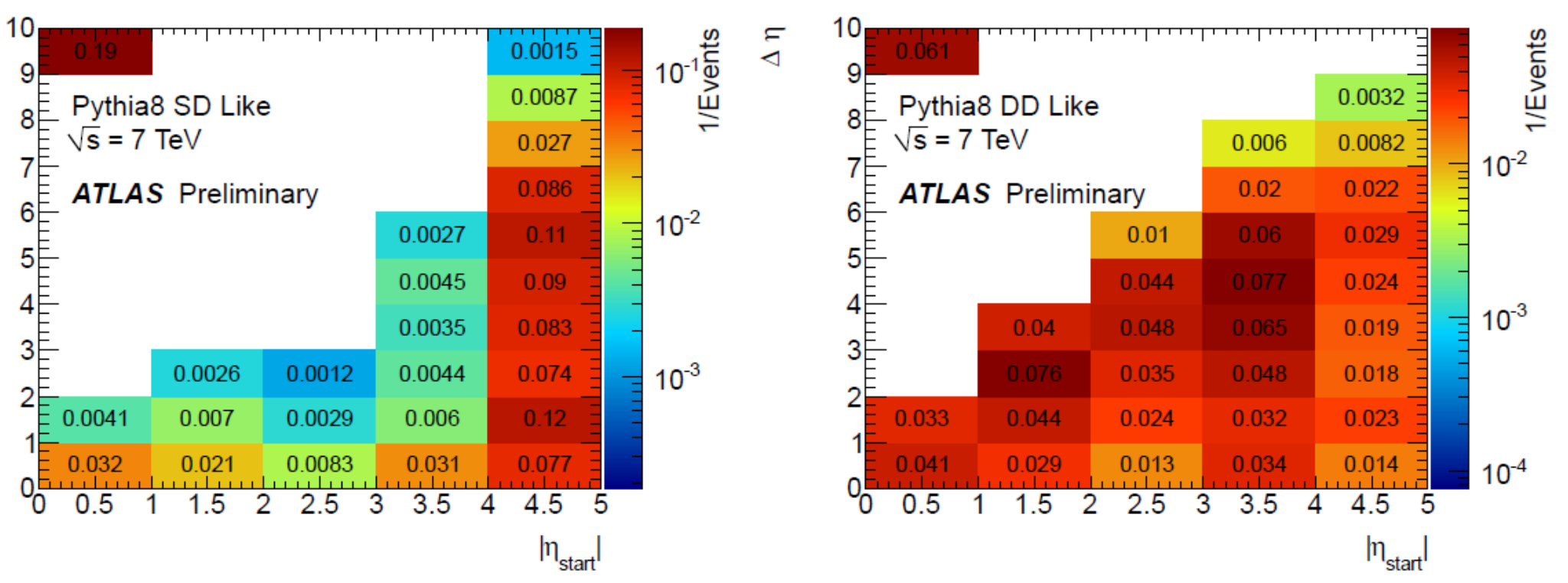

- $\sigma_{\text {inel }}=\sigma_{\mathrm{ND}}+\sigma_{\mathrm{SD}}+\sigma_{\mathrm{DD}}$

- One template for each component.

- Since limited acceptance, re-organization of the event in SD/DD-like events.

- DD events with one dissociated system with $\xi=\mathrm{M}^{2} / \mathrm{s}<5 \times 10^{-6}$ have a SD signature in the apparatus (SD-like events)

- Use this definition in the MC. 


\section{Rapidity Gap Cross Section}
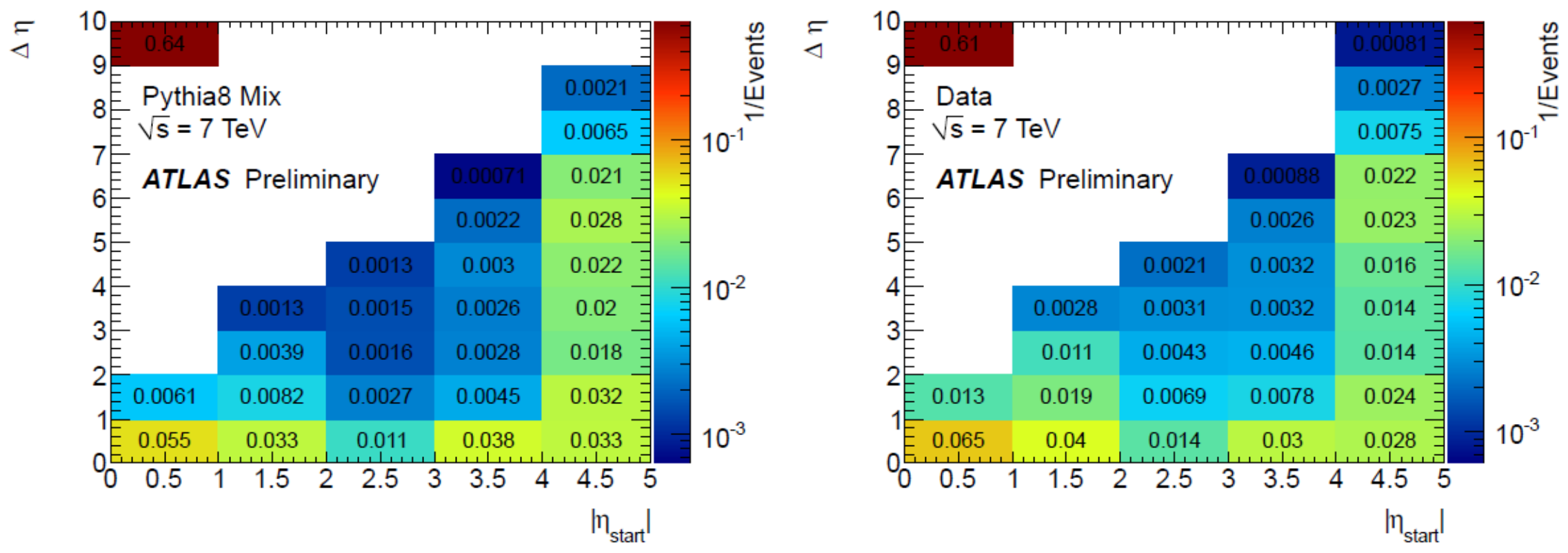

-Fit data-MC for the tuning using the likelihood method.

- In this way the parameter $f_{D}$ (ratio diffractive/total inelastic events) was determined

-It was found for Pythia8 $f_{D}=30 \pm 0.3$ (stat.) \pm 3.8 (sys.) 


\section{Rapidity Gap Cross Section}

Control distribution for MC

Forward rapidity gap distribution (detector level). Acceptable description at large gap size.

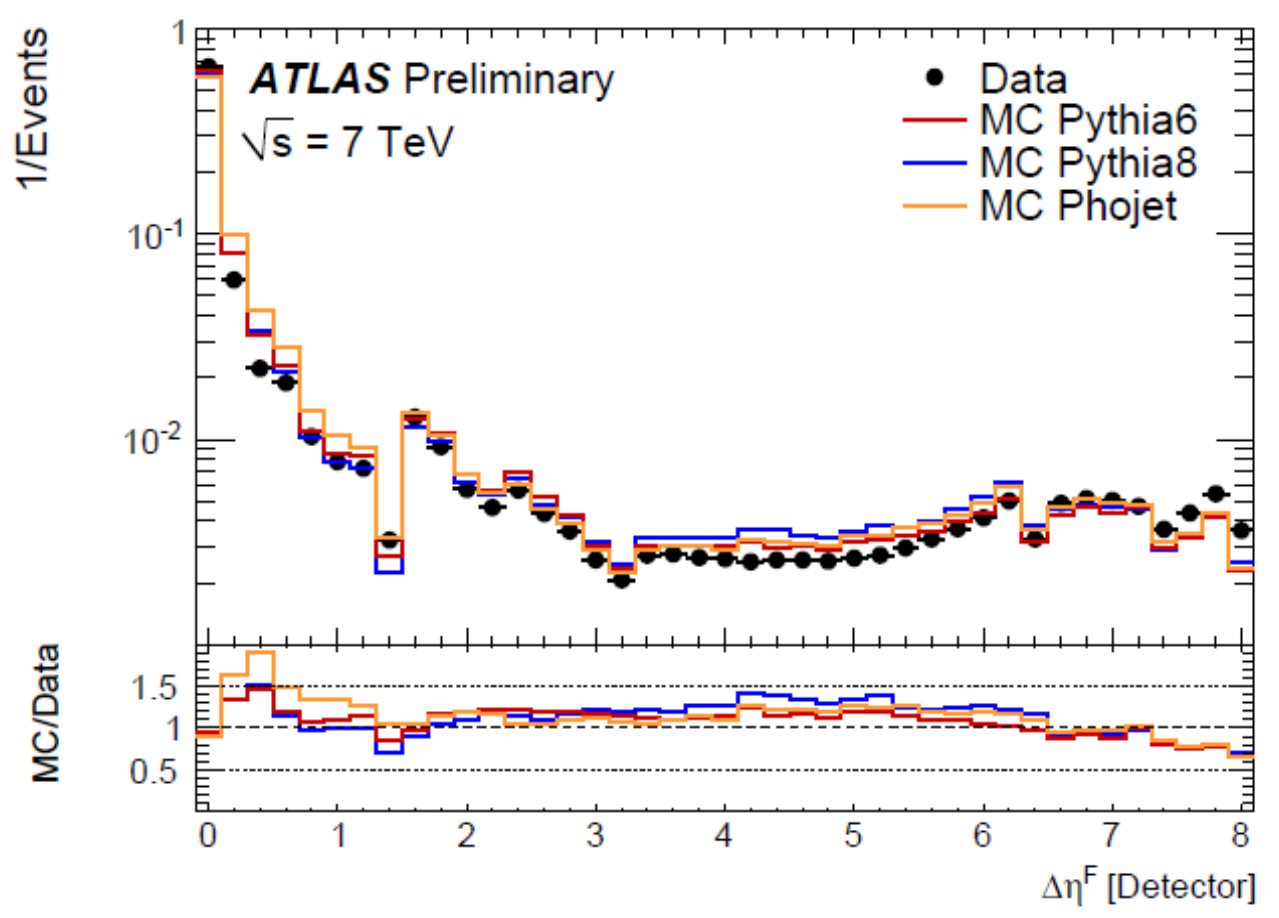

Pythia8 chosen for correcting the data. 


\section{Rapidity Gap Cross Section}

- Measured differential cross section in function of the forward rapidity gaps, together with MC prediction. - Contribution non diffractive events, important for gaps $<2$.

- For forward rapidity gaps > 4 SDlike events dominant.

- Plateau observed $\sim 1 \mathrm{mb}$.

- Data uncertainty typically $20 \%$

- Pythia models give a better description for small rapidity gaps.

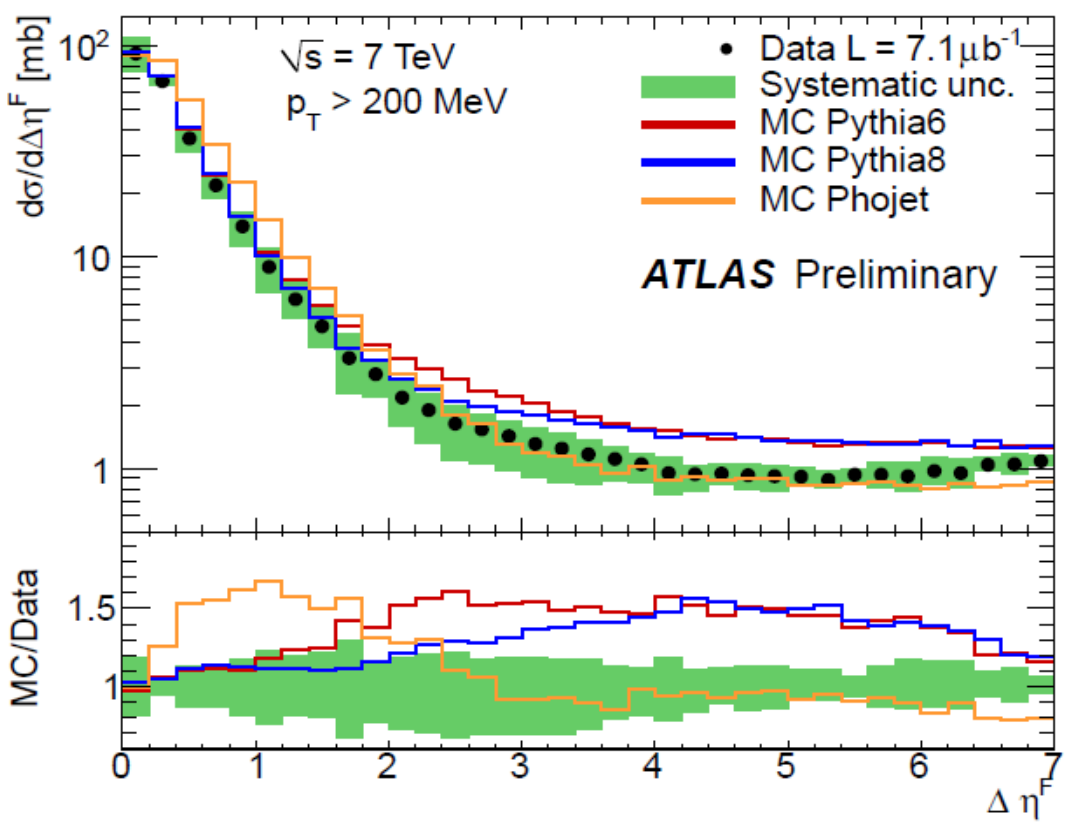
-Phojet successful at large gaps value. 


\section{Rapidity Gap Cross Section}
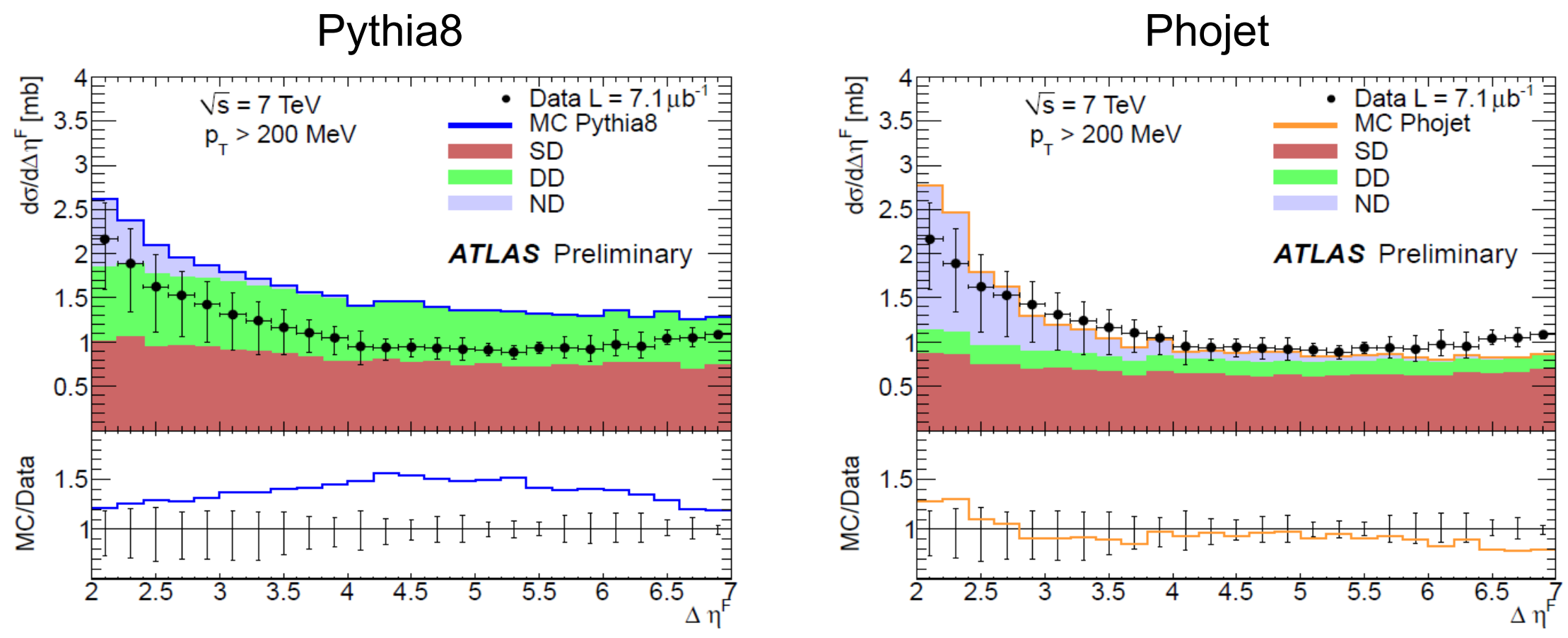

A zoom in the diffractive region and comparison with Pythia8/Phojet $\rightarrow$ Difference is in the DD contribution. 


\section{Conclusions}

- Intense program for the forward physics with the ATLAS detector.

-ALFA detector installed recently, it will take soon data

- Measurement of the total cross section will be performed in September/October.

-Measurement of the inelastic pp cross section

- $\sigma\left(\xi>5 \times 10^{-6}\right)=60.3 \pm 0.05$ (stat.) \pm 0.5 (sys.) \pm 2.1 (lumi.) $\mathrm{mb}$

- Ratio diffractive / inelastic events between $24 \%$ and $30 \%$.

-Rapidity gap studies performed

- $f_{D}=30.2 \pm 0.3$ (stat.) \pm 3.8 (sys.)

- Diffractive cross section for forward rapidity gap $>3.5$ was found to be $\sim 1 \mathrm{mb}$. 


\section{Backup}

28.06.2011 


\section{LUCID: LUminosity Cherenkov Integrating Detector}

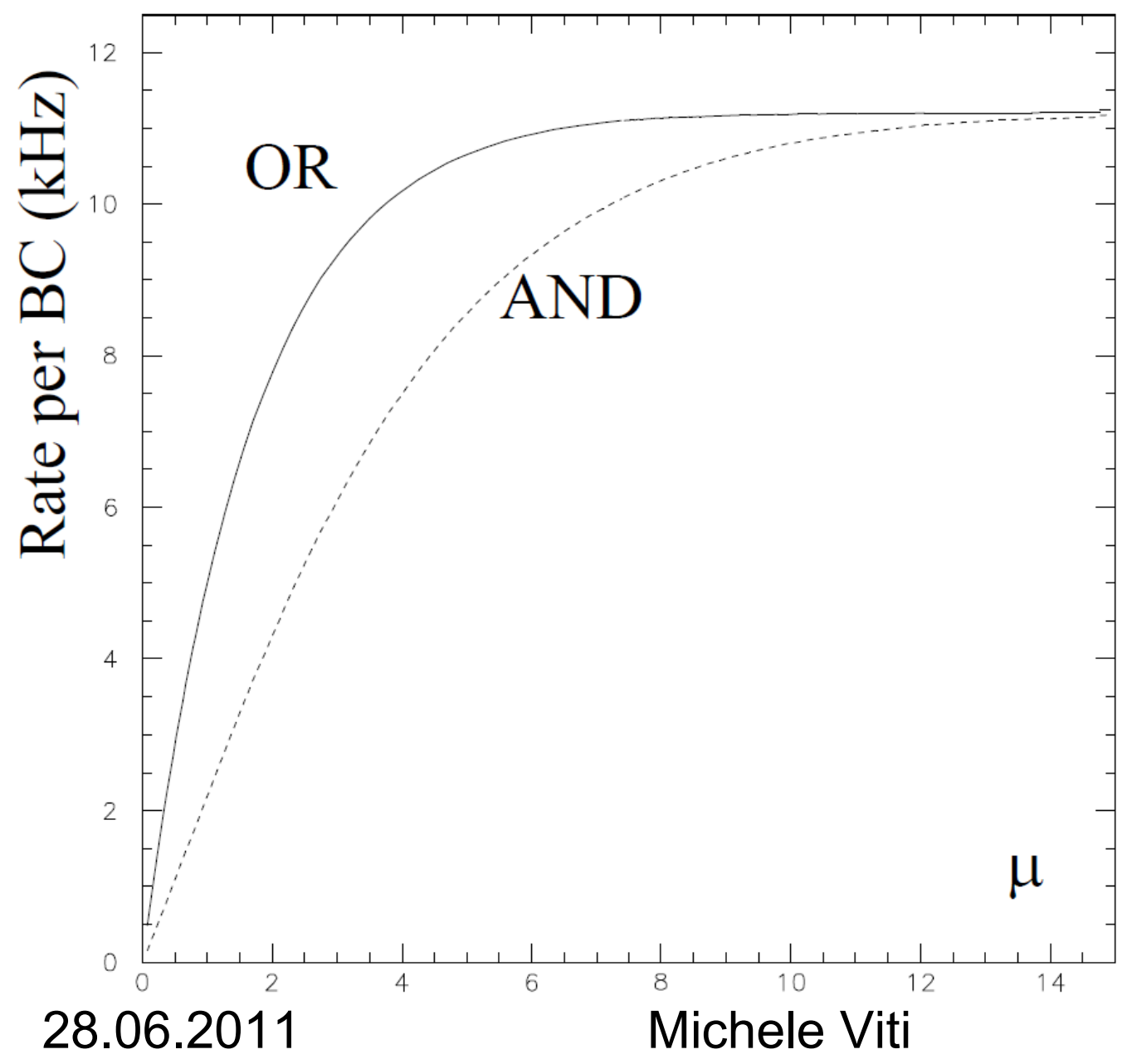




\section{ZDC: Zero Degree Calorimeter}

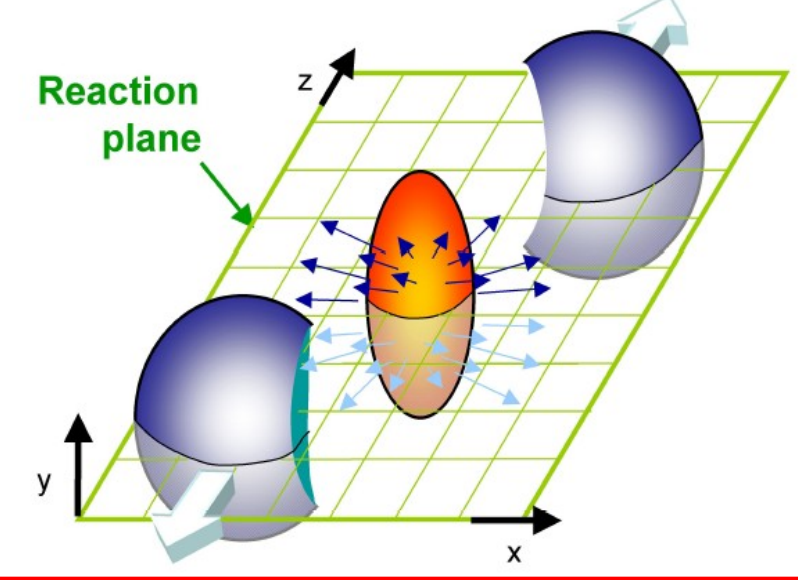

$\mathrm{HI}$ collision

- Minimum bias trigger.

- Information about the collision centrality (number of "spectator neutrons", $20 \%$ energy resolution required).

- Tag hard photon-production and quasi elastic vector meson production (ultra-peripheral collisions).

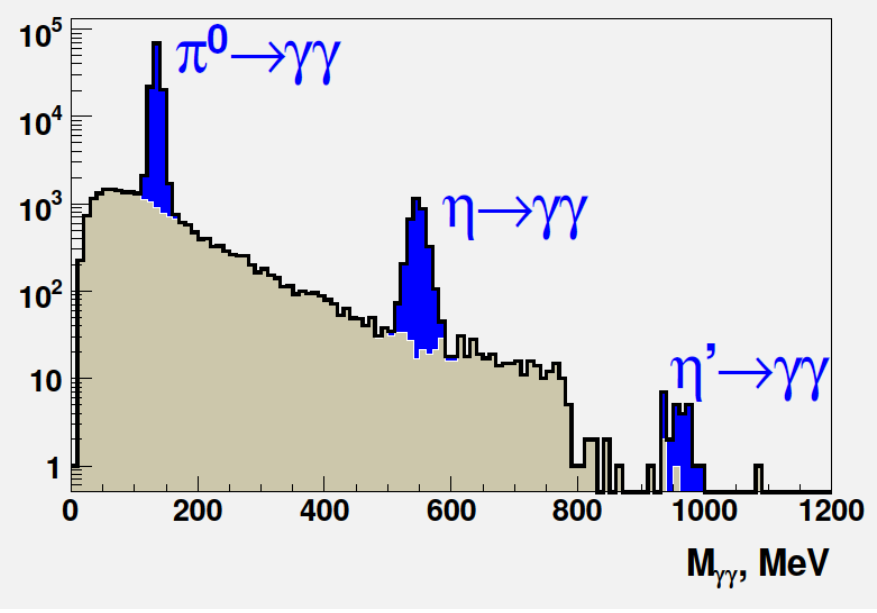

pp collision

- Measurement of many production cross section.

- Luminosity monitor.

- Beam tuning.

- Increase the acceptance of the central detector (important for diffractive processes). 


\section{ZDC: Zero Degree Calorimeter}

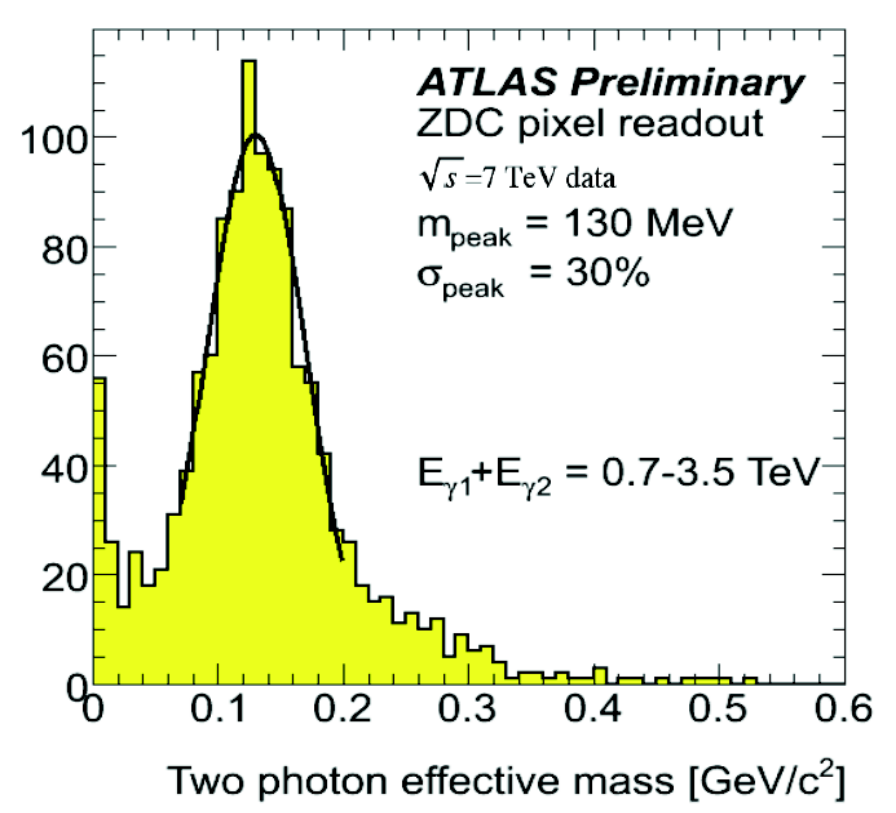

$\pi^{0}$ reconstruction with the first hadronic module for ZDC. The resolution is dominated by the energy resolution.

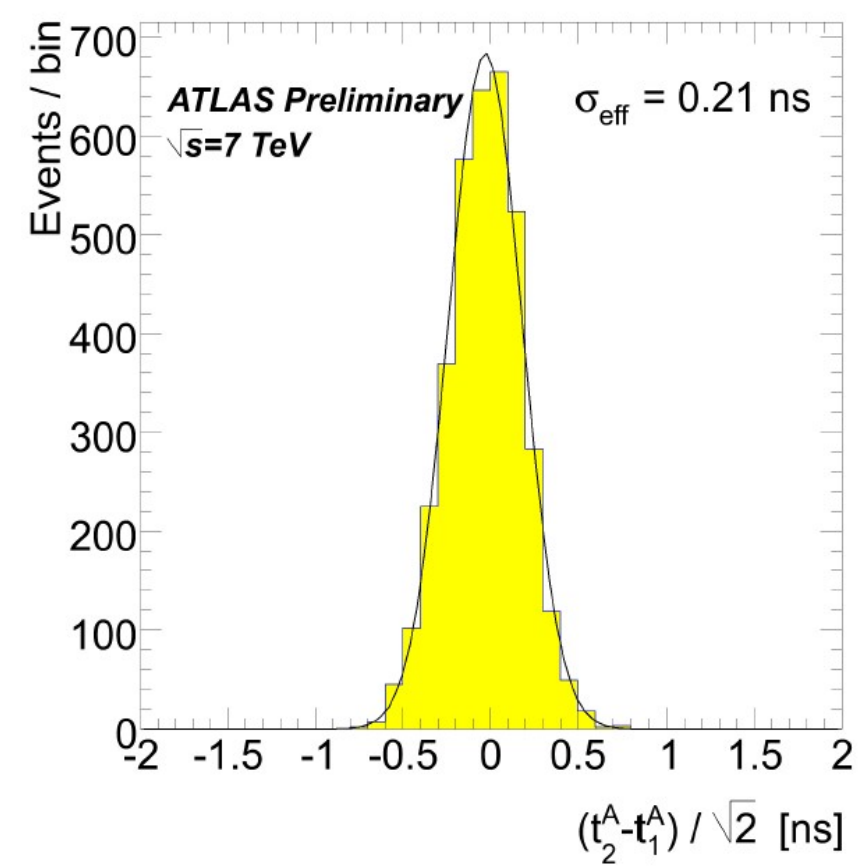

Distribution of events in module 2 time minus that in module 1 in ZDC-A, divided by $\sqrt{ } 2$. 


\section{Luminosity measurement}

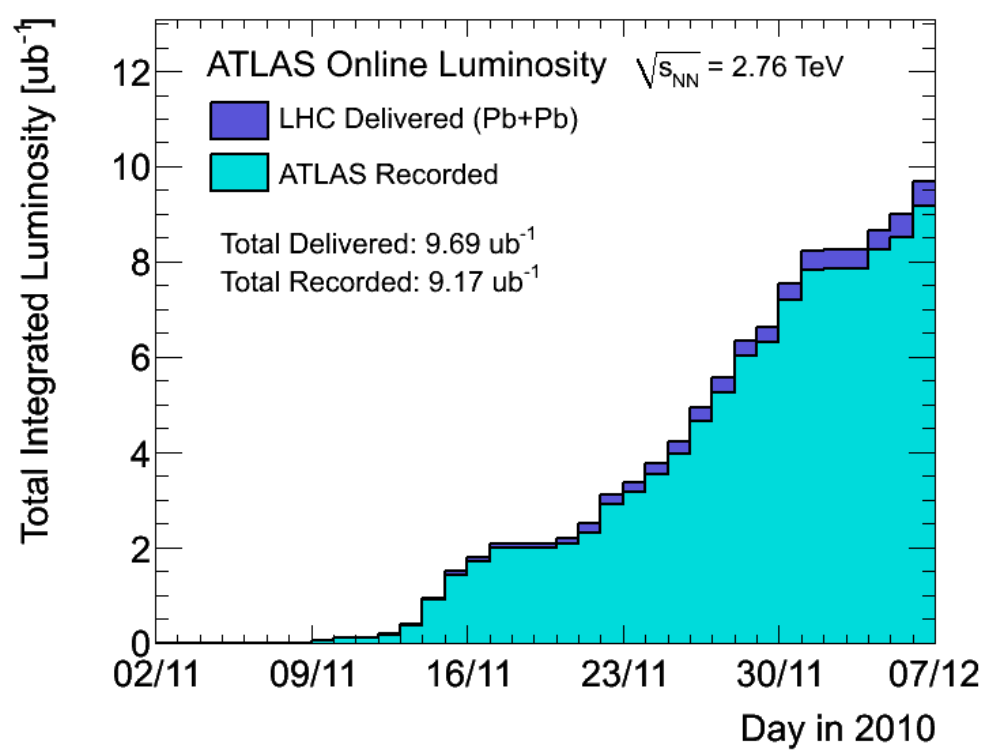

- ATLAS efficiency 95\%

- Max peak lum. 3.04×1025

- Max bunch. 129 


\section{VdM Scan luminosity calibration}

- 5 dedicated runs in 2010 , no physics runs

- Beam displaced $\pm 6 \sigma$, register activity from all luminosity monit

$$
\begin{aligned}
& \text { With the Scan we obtain } \\
& \Sigma_{x, y}=\frac{1}{\sqrt{2 \pi}} \frac{\int R_{x, y}(\delta) d \delta}{R_{x, y}}
\end{aligned}
$$

$R_{x, y}$ is the curve describing the data, this case a double Gaussian $\delta$ separation for $\mathrm{x}, \mathrm{y}$, for a normal Gaussian , trivially $\Sigma_{x, y}=\sigma_{x, y}$
Luminosity calculated trough the formula

$$
L=\frac{f_{r} n_{b} I_{1} I_{2}}{2 \pi \Sigma_{x} \Sigma_{y}}
$$

$n_{b}$ number of bunches

$f_{r}$ frequency revolution

$\Sigma$ Determined by the VdM Scan $I$ bunch current

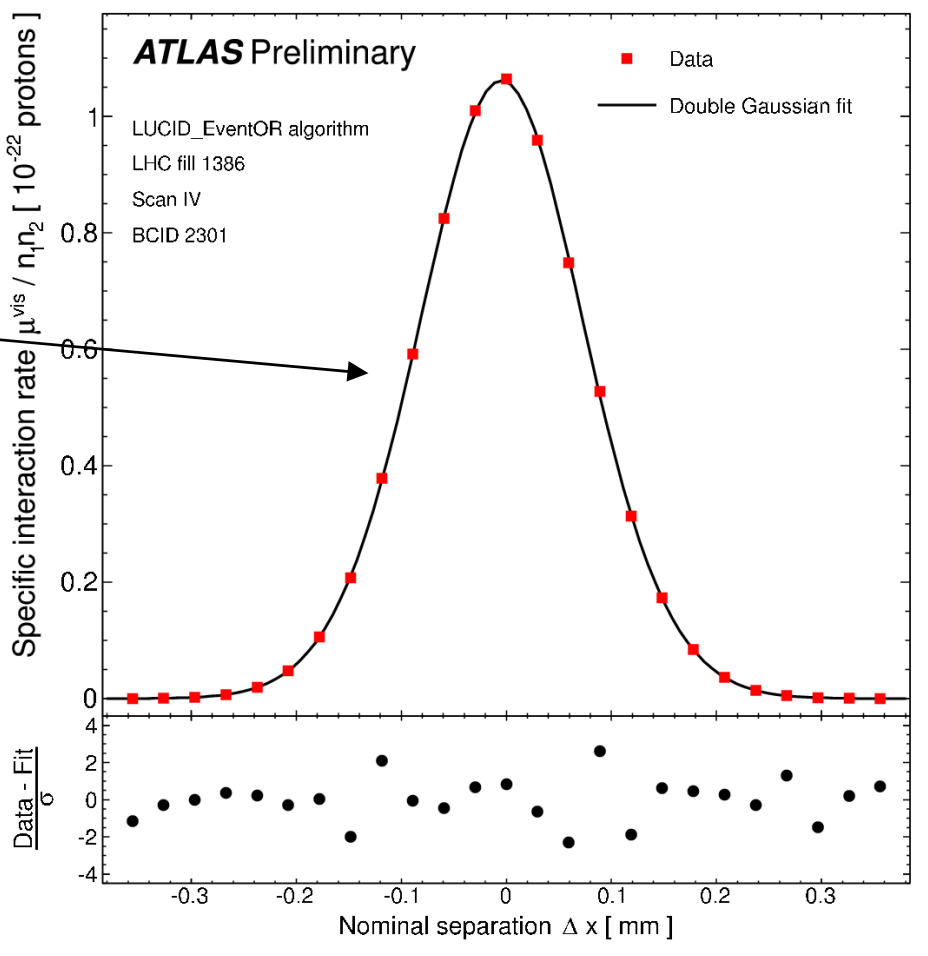

Scans April / Mai $2010 \frac{\delta L}{L}=11 \%$

(Eur.Phys.J.C(2011)71:1630)

Scan October $2010 \frac{\delta L}{L}=3.4 \%$

(ATLAS-CONF - 2011-011)

28.06.2011

Michele 


\section{Forward physics}

- Inelastic cross section $\sim 75 \%$ total cross section.

- Diffractive events are $20-30 \%$ of the inelastic cross section.

- Importance of measurement total/inelastic/elastic cross sections: test of quantum mechanics at high energy.

\begin{tabular}{|c|}
\hline Froissart bound $\sigma_{\text {tot }}<$ Const $\cdot \ln ^{2}(s)$ \\
Optical theorem $\sigma_{t o t} \approx \operatorname{Im} f_{e l}(t=0)$ \\
Dispersion relation $\operatorname{Im} f_{e l}(t=0) \approx \operatorname{Re} f_{e l}(t=0)$
\end{tabular}




\section{Soft/Hard Diffraction}

- Hard processes where one or two protons remains + production of a high $\mathrm{p}_{\mathrm{T}}(|\eta|<2.5)$ central

colorless systems (jets, W,Z).

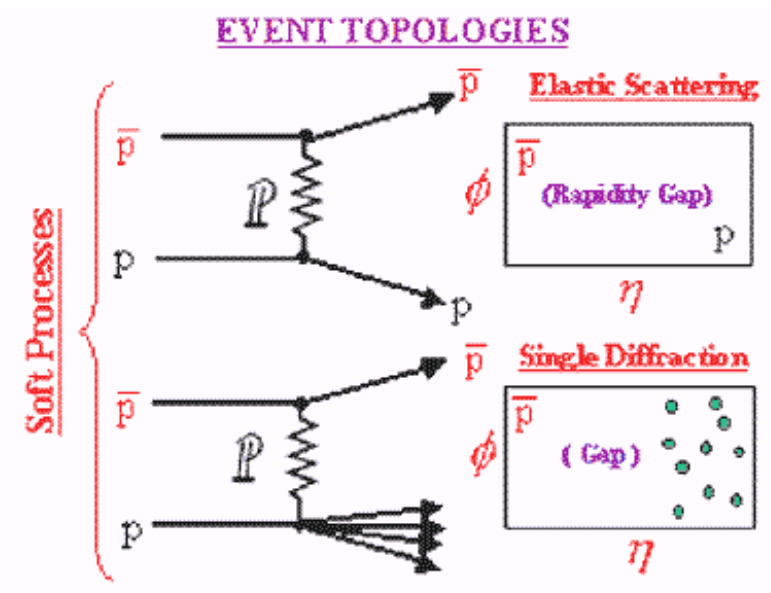

- Central detector needed + trigger (veto) by LUCID, ZDC, MBTS.

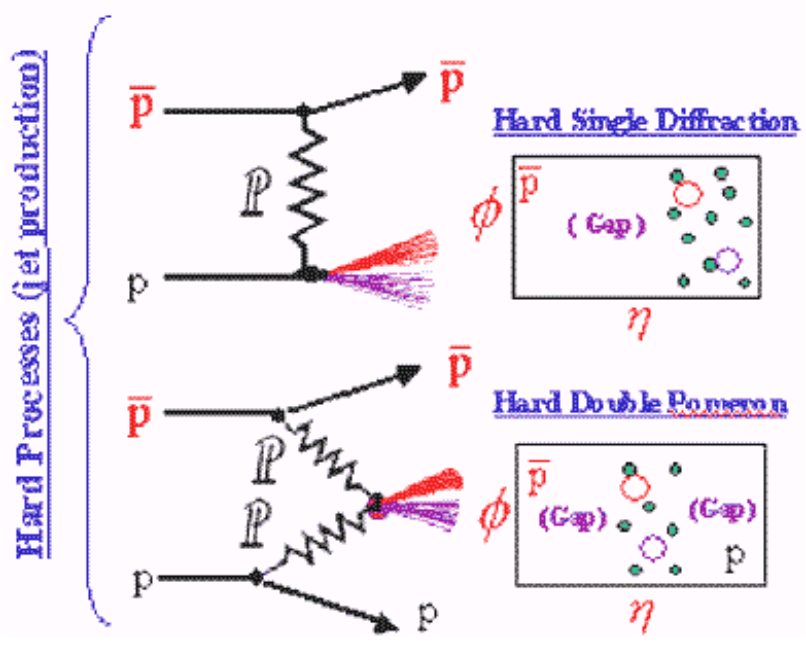




\section{Central Exclusive Production}

- Reaction of the form $p+p \rightarrow$ $\mathrm{p}+\mathrm{X}+\mathrm{p}$.

- $X$ is fully measured and no other particles are produced.

- Tagging the outgoing protons, these processes can provide a clean environment even with high luminosity (large pile-up).

- For the moment, we do not

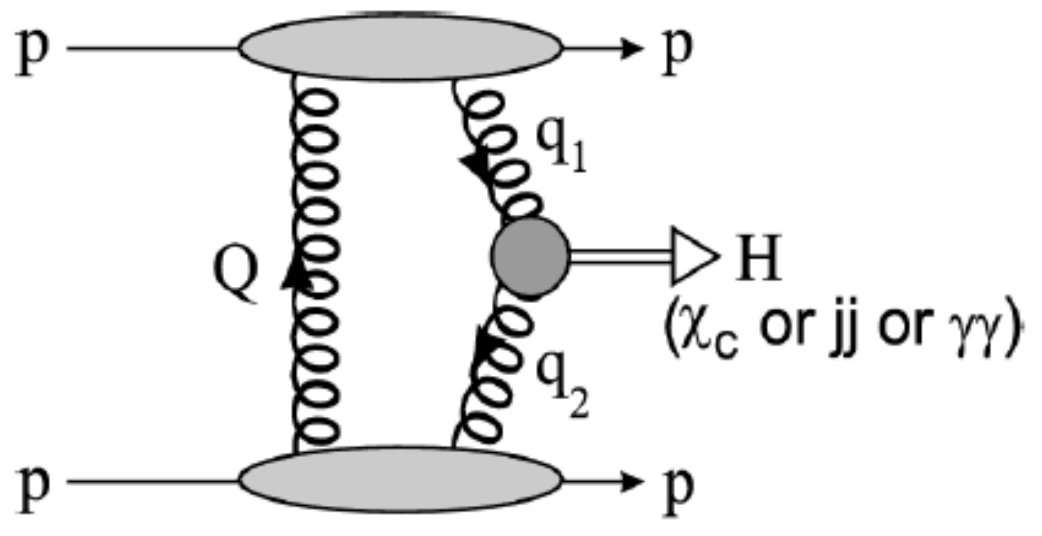
have a dedicated detector for this.

- Possible studies on Dijets,

$\mathrm{W}^{+} \mathrm{W}^{-}$, Higgs and much more... 28.06.2011

Michele Viti 


\section{Soft Single Diffraction with}

\section{ALFA}

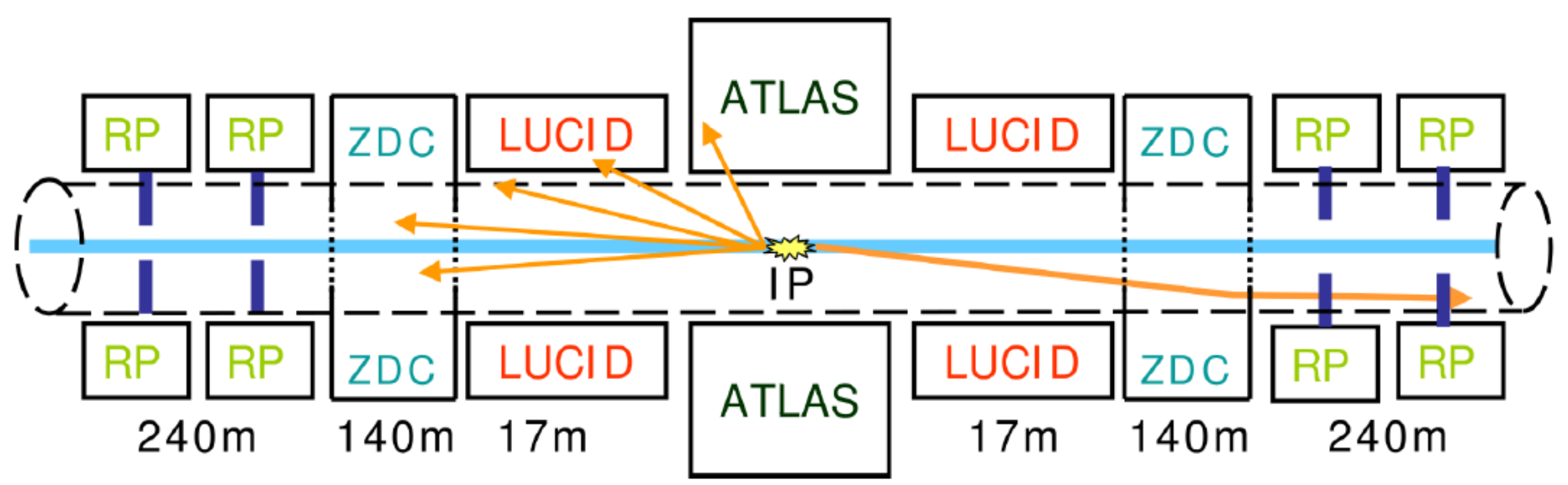

- LUCID/ZDC as trigger.

- ALFA detector to reconstruct the momentum loss $\xi=\left(p-p_{0}\right) / p_{0}$ of the remaining proton.

- Measurement performed at high beta (luminosity runs).

28.06.2011

Michele Viti

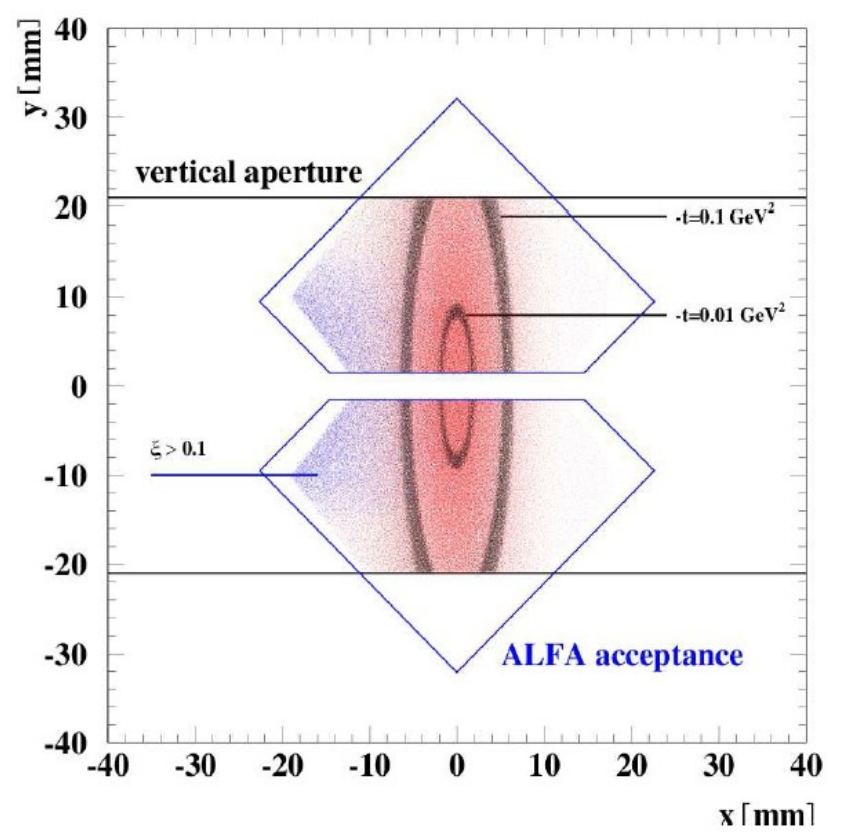




\section{Inelastic pp cross section}

-Fundamental quantity.

- It cannot be calculated with QCD (many approaches...).

-Measurement at lower energy, large uncertainty to extrapolation at higher energy.

-Several models

- $S^{(\alpha-1)}$, where $\alpha$ is the pomeron trajectory intercept.

- Logarithmic behavior.

-Froissart bound: the (inelastic) cross section cannot rise faster than Const $x^{2}(\mathrm{~s})$. 


\section{Rapidity Gap Cross Section}
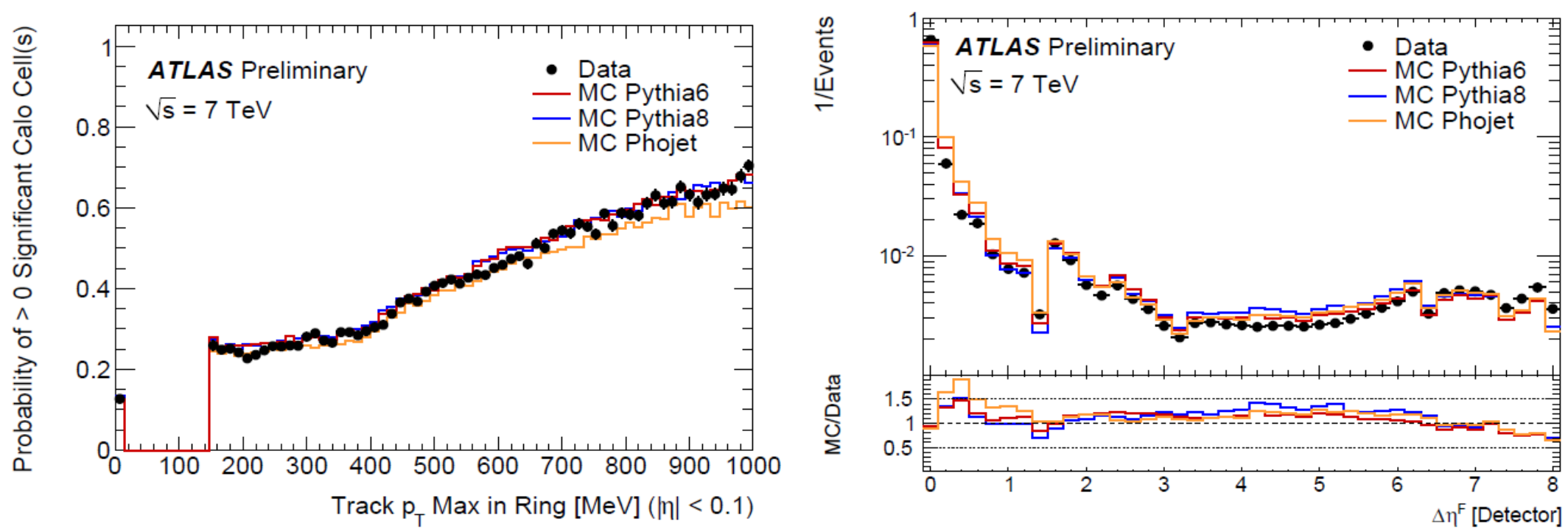

Control distribution for MC

Probability for detecting activity in the most central ring of the calorimeter in function of highest $\mathrm{p}_{\mathrm{T}}$ track.
Forward rapidity gap distribution (detector level). Acceptable description at large gap size.

Pythia8 chosen for correcting the data. 


\section{Rapidity Gap Cross Section}

Formula used for the measurement :

$\frac{\mathrm{d} \sigma\left(\Delta \eta^{F}\right)}{\mathrm{d} \Delta \eta^{F}}=\frac{A\left(\Delta \eta^{F}\right)}{\Delta \eta_{\text {ring }}} \frac{N\left(\Delta \eta^{F}\right)-N_{\mathrm{BG}}\left(\Delta \eta^{F}\right)}{\varepsilon\left(\Delta \eta^{F}\right) \times \mathcal{L}}$
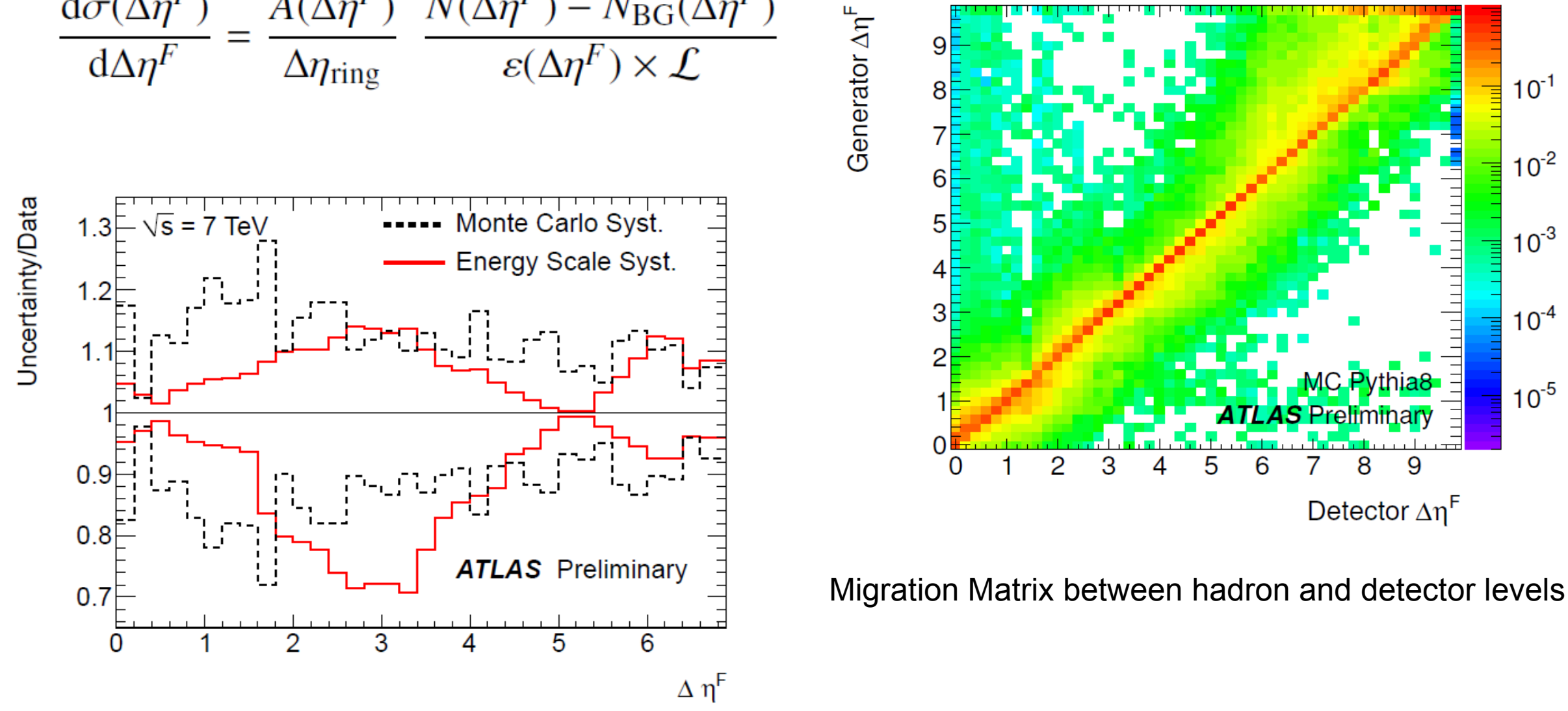

Migration Matrix between hadron and detector levels.

28.06.2011

Michele Viti 